Document downloaded from:

http://hdl.handle.net/10251/38859

This paper must be cited as:

Navarro, P.; Morais Ezquerro, SB.; Gavaldón, JA.; Pérez, AJ.; Puchades Pla, R.; Maquieira Catala, Á. (2013). Arrays on disc for screening and quantification of pollutants. Analytica Chimica Acta. 784:59-64. doi:10.1016/j.aca.2013.04.041.

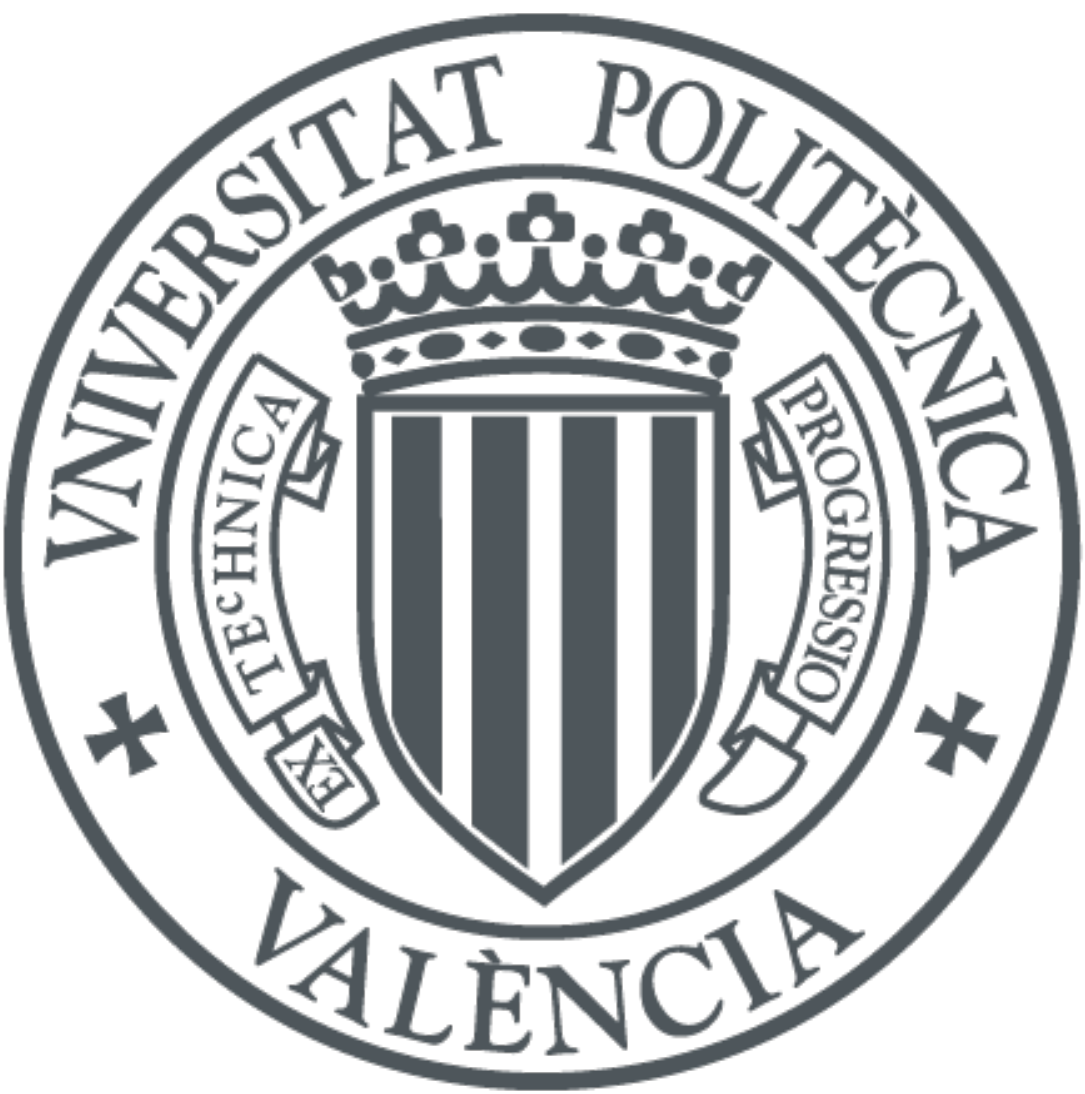

The final publication is available at

http://dx.doi.org/10.1016/j.aca.2013.04.041

Copyright Elsevier Masson

Additional Information 
Document downloaded from:

http://hdl.handle.net/10251/38859

This paper must be cited as:

Navarro, P.; Morais Ezquerro, SB.; Gavaldón, JA.; Pérez, AJ.; Puchades Pla, R.; Maquieira Catala, Á. (2013). Arrays on disc for screening and quantification of pollutants. Analytica Chimica Acta. 784:59-64. doi:10.1016/j.aca.2013.04.041.

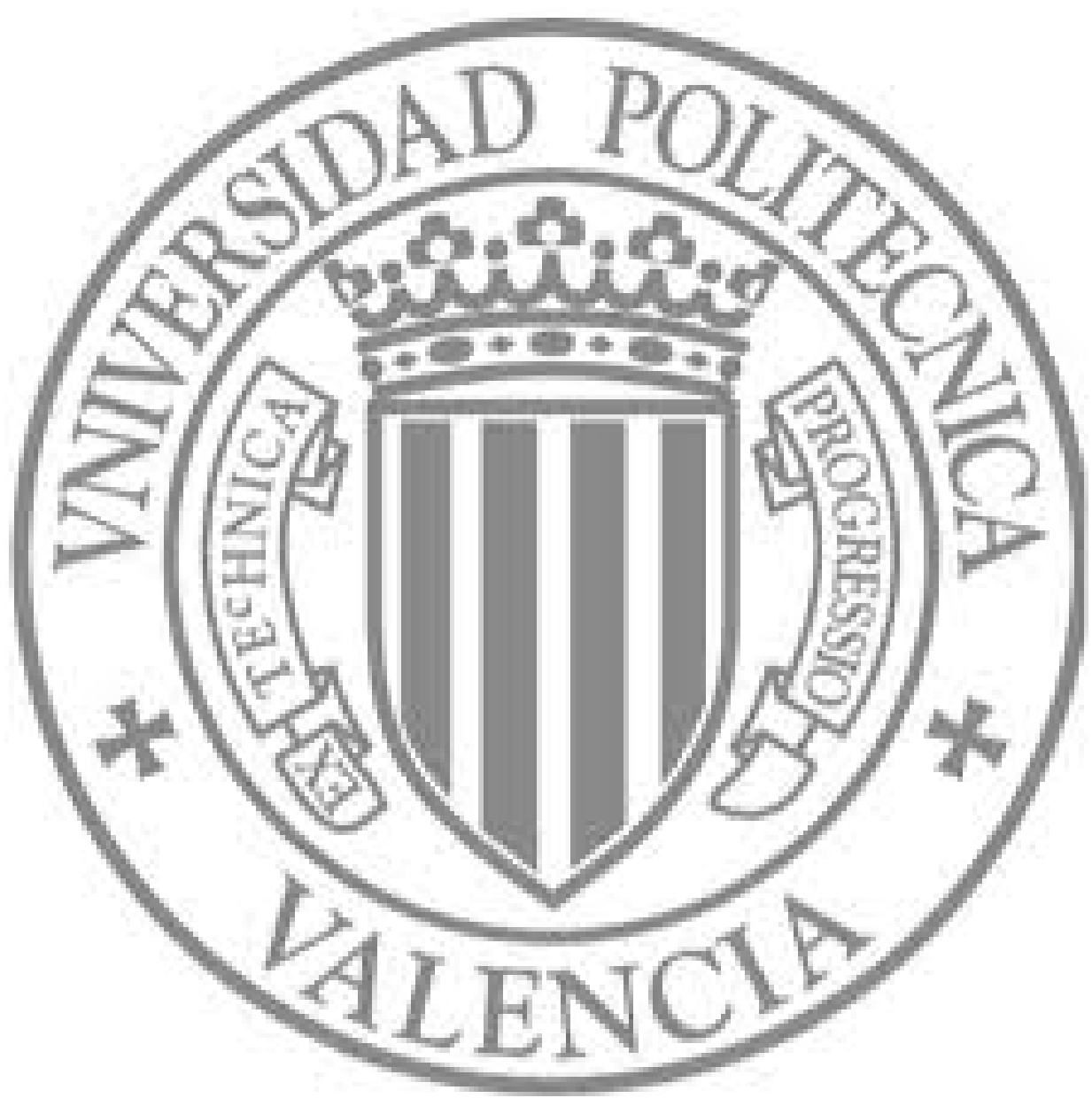

The final publication is available at

http://www.sciencedirect.com/science/article/pii/S0003267013005849/pdfft?md5=b87a1778C

Copyright Elsevier Masson 


\title{
Arrays on disc for screening and quantification of pollutants
}

\author{
Patricia Navarro $^{a}$, Sergi Morais ${ }^{\mathrm{b}, *}$, Jose A. Gabaldón ${ }^{\mathrm{a}, * *}$, Antonio J. Pérez ${ }^{\mathrm{a}}$, \\ Rosa Puchades ${ }^{\mathrm{b}}$, Ángel Maquieira ${ }^{\mathrm{b}}$ \\ a Departamento de Tecnología de la Alimentación y Nutrición, Universidad Católica de San Antonio, Campus de los Jerónimos s/n, 30107-Guadalupe, \\ Murcia, Spain \\ ${ }^{\mathrm{b}}$ Centro de Reconocimiento Molecular y Desarrollo Tecnológico (IDM), Universitat Politècnica de Valencia, Camino de Vera s/n, 46071 Valencia, Spain
}

\section{H I G H L I G H T S}

- Disc-arrays were developed for quantitative determination of pollutants.

- Dozens of samples were analyzed in 40 min after simple dilution.

- High-throughput multiplexed screening of organic contaminants at a very competitive value.

- User-friendly and robust methodology with high applicability for monitoring programs, in situ analysis, in low-level labs or outside the lab setting.

\section{A R T I C L E I N F O}

Article history:

Received 9 November 2012

Received in revised form 15 April 2013

Accepted 24 April 2013

Available online 1 May 2013

\section{Keywords:}

Arrays

Compact discs

Immunoanalysis

Pollutants

\section{G R A P H I C A L A B S T R A C T}

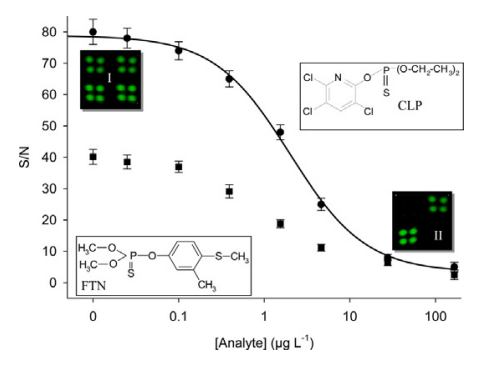

\begin{abstract}
A B S T R A C T
A rapid compact disc based methodology for screening and quantification of organic pollutants in mandarin juices is presented. The assay is established on the coating conjugate indirect competitive principle and developed under disc-array configuration. The detection is based on the acquisition of attenuated reflective signals that were proportional to optical density of the immunoreaction product. The competitive assay is applied to quantify simultaneously, in a selective manner, non-systemic insecticides in mandarin juices. The detection limits were 0.2 and $0.1 \mu \mathrm{gL}^{-1}$ and the sensitivity 2.1 and $1.5 \mu \mathrm{g} \mathrm{L}^{-1}$, for chlorpyrifos and fenthion, respectively. Pollutants were directly quantified after sample dilution in a total time of $40 \mathrm{~min}$. Also, the implementation of positive and negative controls into the array configuration served as an automatic quality control test. The effect of thermal treatment on pesticide dissipation was studied and found that it was insignificant under the studied conditions. Recovery intervals ranged from $96-105 \%$ to $94-103 \%$, for chlorpyrifos and fenthion, respectively and were similar to those obtained with gas chromatography coupled to mass spectrometry. In the current configuration, 64 samples can be simultaneously analyzed on a disc at a very competitive value, demonstrating its potential for highthroughput multiplexed screening applications for controlled monitoring programs in low-level labs or outside the lab setting.
\end{abstract}

() 2013 Elsevier B.V. All rights reserved.

\footnotetext{
* Corresponding author. Tel.: +34 963877342.

** Corresponding author.

E-mail addresses: smorais@qim.upv.es (S. Morais), jagabaldon@pdi.ucam.edu (J.A. Gabaldón).
}

\section{Introduction}

According to the National Research Council (NRC) reports, dietary intake represents the major cause of organic pollutants exposure for infants and children [1], and this could produce a serious health risk for this population group. In order to ensure 
that this type of pollutants do not move into food chain, the European Food Safety Authority (EFSA) assesses the dietary intake of them at pre- and post-regulation level. In this context, the European Commission has proposed extensive food control programs that basically define maximum residue limits (MRLs) and highest residue measured (HRM) to establish the lowest consumption threshold [2]. Fruit juices that are manufactured as pasteurized and concentrated beverages are the most frequent source of diary fresh fruit intake [3]. Due to the rigorous legislations regulating the allowable amount of contaminants in fresh and manufactured beverages, the presence, in particular, of pesticide residues in commercial juice is one of the problems faced by the citrus sector since also it becomes an obstacle to free competition within the European Union and sea side trade. Therefore, there is an increasing demand on food manufacturers and farmers to ensure safe food supplies.

The reference methodologies for the determination of pesticide residues employ sophisticated and expensive instrumentation such as gas or liquid chromatography coupled with different detectors [4-6] and requires tedious sample pretreatments, making these methodologies unfeasible for rapid, cost-effective and in situ analysis. For this reason, food industries demand for more portable, fast and cheap technologies to simplify the operational procedures and increase the number of analysis. In this line, immunoanalytical tools are easily adaptable to different formats and analytical scenarios, allowing for simplification of sample preparation and representing cost-effective and simpler methodologies [7-9]. An example of this type of analytical methodology is represented by lateral flow immunochromatographic assays that remain the current preferred option in terms of rapidity for point-of-care screening and in situ analysis [10-14]. In most of the cases, however, this type of technology analyzes one analyte in one strip, giving qualitative visual readouts and resulting expensive for screening applications. The scaling-up of the methodology is also a tough topic and it is not commonly approached due to limited sensing surface. Also, the feasibility of adapting the manufacturing process to a resource-limited setting remains challenging.

In the search for more powerful analytical tools, planar microarray technology go beyond since it can be easily adapted to high throughput tests, show multiplex capabilities and allow quantitative analysis with high potential applicability in life sciences [15-17]. In this line, arrays on compact disks have shown great potential when they are applied in the areas of food safety and environmental analysis $[18,19]$. Arrays on disc show high working capacity and present the additional advantage of being scanned by disc drives. Besides, standard disc drives are commercially available at very low price, offering robustness and portability. These characteristics make this technology a suitable tool for quantitative analysis for further use in industries and in coordinated control programs. For these reasons, the aim of this study was to develop a rapid and sensitive analytical methodology based on multiplexed arrays on commercial discs (DVD-R) for the simultaneous determination of organic pollutants using a standard DVD drive. As a proof of concept, the methodology has been applied to the simultaneous quantification of chlorpyrifos and fenthion in mandarin juices. These non-systemic insecticides, commonly used to control different pests on foliage in fruit, were chosen as model systems to test the capabilities of the arrays on disc to determine pesticide residues in mandarin juices. The results were validated in terms of sensitivity, selectivity, precision and robustness by gas chromatography coupled to mass spectrometry (GC-MS). The performances shown by arrays on discs might be of great interest in routine testing for food safety and quality control in monitoring programs and for industries with low equipment settings.

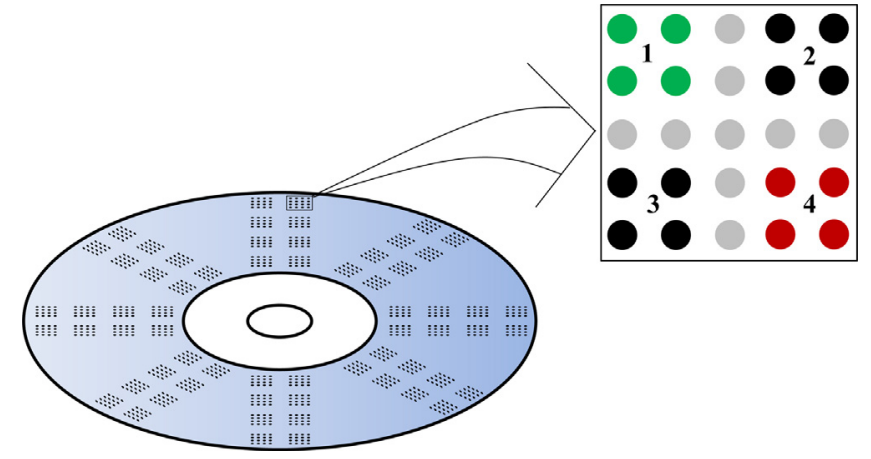

Fig. 1. Layout of arrays on DVD. The disk holds 64 arrays of $5 \times 5$ spots, organized in four blocks of $2 \times 2$ spots and 9 negative spots (in gray), simulating a virtual cross. Blocks correspond to fenthion (1; spots in green) and chlorpyrifos (4; spots in red) systems, BSA (2) and RIgG (3) as positive controls (spots in black), respectively. (For interpretation of the references to color in this figure legend, the reader is referred to the web version of this article.)

\section{Experimental}

\subsection{Chemicals}

Standard chlorpyrifos and fenthion were purchased from CibaGeigy (Barcelona, Spain). Bovine serum albumin (BSA), ovalbumin (OVA), gold labeled goat anti-rabbit immunoglobulins (GAR-Au) and silver enhancer solutions (A and B) were from Sigma-Aldrich (Madrid, Spain). Printing buffer was $0.1 \mathrm{M}$ sodium carbonate buffer, pH 9.6, assay buffer was $10 \mathrm{mM}$ sodium phosphate buffer, $0.05 \%$ Tween 20, pH 7.5 (PBST) and both were filtered through a $0.22 \mu \mathrm{m}$ pore size disk before use.

\subsection{Microarraying}

DVD-R disks were purchased from MPO Ibérica (Madrid, Spain). The discs were first conditioned by gentle ethanol washing, water rinsing, and dried by centrifugation. The coating conjugates OVAC5 and OVA-F1 [20] used for the determination of chlorpyrifos and fenthion, respectively, were diluted in printing buffer and placed into a 384-well plate to dispense $25 \mathrm{~nL}$ of solution onto the disc with a noncontact printing device (AD 1500 BioDot, Inc., Irvine, CA) in microarray format. The disk layout (Fig. 1) consisted in a total of 64 microarrays arranged in blocks, each one of $5 \times 5$ spots. Within each microarray, the blocks correspond to chlorpyrifos and fenthion systems, two positive and several negative spot controls. In this configuration, spots are $500 \mu \mathrm{m}$ in diameter with a track pitch (center to center distance) of $1.0 \mathrm{~mm}$, achieving an array density of $4.0 \mathrm{spot} / \mathrm{mm}^{2}$. Discs were printed on the polycarbonate surface under controlled temperature and relative humidity $\left(25^{\circ} \mathrm{C}\right.$ and $90 \%$, respectively).

\subsection{Microimmunoassay protocol}

The assays on DVD-arrays were based on an indirect competitive format. First, coating conjugate (OVA-C5 for chlorpyrifos and OVA-F1 for fenthion), BSA ( $\left.25 \mathrm{mg} \mathrm{L}^{-1}\right)$ and nonimmunized rabbit sera (RIgG) (1/1000 dilution) solutions were arrayed onto the polycarbonate surface of the disc. BSA and RIgG were used as positive controls for the first and second immunoreaction steps, respectively, and ovalbumin solution $\left(50 \mathrm{mg} \mathrm{L}^{-1}\right)$ was included as a negative control. After $16 \mathrm{~h}$ at $4{ }^{\circ} \mathrm{C}$, the disk was thoroughly washed with PBST, rinsed with deionized water, and dried by centrifugation at $800 \mathrm{rpm}$. For single-target assay optimization, $12 \mu \mathrm{L}$ of polyclonal sera [20] (1/1000 dilution for C2-II and 1/500 for F7-I) in PBST, with or without analyte, was dispensed over the array using a 
micropipette. After 15 min incubation at room temperature the disk was washed with PBST buffer and then rinsed with deionized water. Next, $1.0 \mathrm{~mL}$ of gold-labeled secondary antibody solution (1/50 in PBST) was dispensed onto the disk, and it was evenly distributed along with the whole disc surface with a $12 \mathrm{~cm}$ diameter $0.6 \mathrm{~mm}$ thick dummy plastic disc. After $15 \mathrm{~min}$ at room temperature, the cover surface was removed, and the disk washed and dried as before. The immunoreaction was developed by homogenously distributing $1.0 \mathrm{~mL}$ of silver enhancer solution onto the disk as before, and the reaction was stopped by washing the disk with water after $8 \mathrm{~min}$. For multiplexed assays, $12 \mu \mathrm{L}$ of a solution prepared with $5 \mu \mathrm{L}$ of PBST or sample with and without analyte, conditioned with $40 \mu \mathrm{L}$ of PBST with $5 \mu \mathrm{L}$ of the mixed sera solution (cocktail) were dispensed on to disc, covering the whole array. The total volume of $50 \mu \mathrm{L}$ was used to analyze samples per triplicate. Next, the immunoassay was performed as described above. The total assay took $40 \mathrm{~min}$. Juice samples were spiked with chlorpyrifos and fenthion within the working range and diluted 1/10 in PBST before analysis. Also, mandarin juice without spiking was used as blank sample.

To evaluate the selectivity of the duplex assay, different chemically related and unrelated compounds were tested. Three calibration curves for each compound were obtained in the optimized working conditions. The cross-reactivity was calculated by comparing the assays response at the midpoint $\left(\mathrm{IC}_{50}\right)$ and expressed as a percentage.

For quantitative determination, discs were read by the prototype as previously described [21]. Basically, the DVD drive (LG Electronics Inc., Englewood Cliffs, NJ), was controlled by custom software (Diskpick), running on a personal computer and connected to it through a USB2.0 universal serial bus interface. During the DVD scanning, the laser hits the immunoreaction products that modify the reflection properties of the DVD surface, attenuating the laser beam intensity that reaches the photodiode of the pickup. The analog signals were directly acquired from the photodiode of the DVD drive and related to optical density of the immunereaction product. For the indirect competitive based immunoassay, the optical density is inversely proportional to analyte concentration. Inhibition curves were mathematically analyzed by fitting experimental results to a sigmoidal four-parameter logistic equation.

\subsection{Instrumentation and chromatographic conditions}

GC-MS analyses were performed in a 6890 GC gas chromatography coupled to a 5973 MS system (Agilent Technologies, Palo Alto, CA) with electron impact ionization detection. A HP5-MS capillary column (Agilent 19091S 433E) with a $0.25 \mathrm{~mm}$ i.d., $30 \mathrm{~m}$ and $0.25 \mu \mathrm{m}$ phase film diameter was used for the determination of organophosphorous pesticides. The pollutants were extracted from $100 \mathrm{~mL}$ of mandarin juice samples using magnetic stir bars ( $20 \mathrm{~mm}$ length $\times 0.5 \mathrm{~mm}$ film thickness) coated with poly(dimethylsiloxane) (PDMS). The bars were previously conditioned in an empty thermal desorption tube at $300^{\circ} \mathrm{C}$ for $4 \mathrm{~h}$ with a helium flow rate of $50 \mathrm{~mL} \mathrm{~min}{ }^{-1}$. After stirring the sample for $24 \mathrm{~h}$ at $900 \mathrm{rpm}$, the bars were thermally desorbed in the splitless mode at $280^{\circ} \mathrm{C}$ for $6 \mathrm{~min}$, transferring the analyte to the PTV injector at a $75 \mathrm{~mL} \mathrm{~min}^{-1}$ helium flow rate. Sodium chloride $(20 \%$, $\mathrm{w} / \mathrm{v}$ ) was used as the matrix modificator. The temperature program was $2 \mathrm{~min}$ at $70^{\circ} \mathrm{C}$ then, increased to $200^{\circ} \mathrm{C}$ at a rate of $30^{\circ} \mathrm{C} \mathrm{min}-1$, hold for $1 \mathrm{~min}$, and increased to $280^{\circ} \mathrm{C}$ at $3^{\circ} \mathrm{C} \mathrm{min}^{-1}$, with a final isothermal period of $2 \mathrm{~min}$. The PTV injector and interface temperatures were held at $280^{\circ} \mathrm{C}$. The detection was made in the full scan mode- $m / z$ range $50-400$ and for confirmation of the spectral data, the characteristic ions for chlorpyrifos $(\mathrm{m} / \mathrm{z}$
$314,199,197,97)$ and fenthion $(m / z 278,169,125,109)$ were used.

\section{Results and discussion}

\subsection{Single-target assays}

The majority of reported bioanalytical based screening methods for pollutants analyze one analyte at one time. In our study, we design arrays on discs with the goal of using it as high-throughput analysis system and for simultaneous determination of pollutants residues. For that, the disc included 64 arrays arranged in four blocks of $2 \times 2$ spots. Two blocks were specifically used for CLP and FTN quantification, whereas the rest of the blocks served as automatic quality controls (see Fig. 1).

In order to demonstrate the multianalyte capability, previously developed immunoreagents were used to set up the methodology on DVDs. For that, the selected [20] coating conjugate/sera pairs were used for developing the arrays on DVDs. As far as optimization is concerned, a wide interval of coating conjugate concentration (ranging from 0.10 to $50 \mathrm{mg} \mathrm{L}^{-1}$ ) was tested against serial dilutions of polyclonal sera (1/500-1/50.000 dilution) C2II and F7-I for CLP and FTN assays, respectively. Optimal coating conjugate concentrations and sera dilutions were selected on the basis of obtaining good signal intensity $(\mathrm{S} / \mathrm{N}>35)$ and reproducibility $(<15 \%)$ by check-board titration in a competitive format. The optimum concentration was found to be $25 \mathrm{mg} \mathrm{L}^{-1}$ for OVA-C5 and $40 \mathrm{mg} \mathrm{L}^{-1}$ for OVA-F1 and $1 / 1000$ and $1 / 500$ sera dilution for C2-II and F7-I, respectively. Regarding detection antibody, the optimal concentration was obtained diluting the gold labeled secondary antibody (GAR-Au) $1 / 50$ fold in PBST.

Selectivity studies were performed under the optimal immunoreagent concentrations and found that single-target systems were selective, since each serum only recognized its specific coating conjugate. Signal-to-noise ratio $(\mathrm{S} / \mathrm{N})$ values were $76.4 \pm 4.8$ and $37.2 \pm 2.9$ for single-target CLP and FTN assays, respectively. In contrast, nonspecific recognition was negligible showing $\mathrm{S} / \mathrm{N}$ values below the limit of quantification $(\mathrm{S} / \mathrm{N}<5)$. These results are at the same level of selectivity as those reported by the respective ELISA plate methods. In a second set of experiments, cross-reactivity studies were performed by measuring signal intensity variation to the presence of high analyte concentrations $\left(>10 \mathrm{mg} \mathrm{L}^{-1}\right)$. In these experiments, single-target assays were carried out and cross-reactivity calculated as percentage of inhibition. In all cases, signal variation was below $0.01 \%$, indicating high selectivity to analyte solutions. This fact is essential when considering single assay candidates to be applied in multiplexed analysis. Furthermore, the selectivity of the assay on disc was evaluated using a set of organophosphorus insecticides and metabolites because of their similar structure to chlorpyrifos and fenthion and several non-chemically related pesticides due to their widespread agricultural and domestic use. As shown in Table 1, none of the tested pesticides and metabolites did interfere with the detection of chlorpyrifos and fenthion. Concretely, chlorpyrifos methyl (10\%) and fenchlorphos (3.5\%) were the only related compounds that may interfere with CLP assay and malathion (5.3\%), fenitrothion (3.8\%), diazinon (2.2\%) and fenthion-sulfoxide (1.6\%) with FTN quantification. These results indicate that the methodology based on arrays on disc is highly selective for the simultaneous detection of chlorpyrifos and fenthion. It is also interesting to note that the use of specific antibodies against other pesticides or metabolites or employing generic antibodies could spread the spectrum of targets.

In the disc-array configuration, negative controls served for checking for non-specific signals, whereas positive controls 
Table 1

Cross-reactivity (\%) of the arrays on disc.

\begin{tabular}{lcc}
\hline \multirow{2}{*}{ Compound } & Array & \\
\cline { 2 - 3 } & Chlorpyrifos & Fenthion \\
\hline Chlorpyrifos & 100 & 1.0 \\
Chlorpyrifos-methyl & 10 & $<0.1$ \\
Fenchlorphos & 3.5 & $<0.1$ \\
Chlorpyrifos-oxon & $<0.1$ & $<0.1$ \\
Fenthion & $<0.1$ & 100 \\
Malathion & $<0.1$ & 5.3 \\
Fenitrothion & $<0.1$ & 3.8 \\
Diazinon & 0.2 & 2.2 \\
Fenthion-sulfoxide & $<0.1$ & 1.6 \\
Dichlorvos & $<0.1$ & $<0.1$ \\
Glyphosate & $<0.1$ & $<0.1$ \\
Atrazine & $<0.1$ & $<0.1$ \\
Bromophos & $<0.1$ & $<0.1$ \\
Trichlorpyr & 0.2 & $<0.1$ \\
Trichloronate & $<0.1$ & $<0.1$ \\
\hline
\end{tabular}

were included to normalize assay intra and inter assay signals. For instance, disk area variations that might cause a slight lower/higher-than-normal reading at the controls would have the same effect on the pesticides array block. The signal intensity for the positive controls $(\mathrm{S} / \mathrm{N}=85-88$ ) was constant to different analyte concentrations. Also, inter and intra disc reproducibility, measured as relative standard deviation (RSD) was below $9 \%$ and $7 \%$, respectively, demonstrating the applicability of positive controls for signal normalization.

The sample volume was also another parameter studied. For these experiments, four volumes $(6,12,18$, and $20 \mu \mathrm{L})$ were compared in terms of signal intensity and reproducibility. For the blanks (absence of analyte), signals were significantly similar for all the tested volumes, varying from 10,870 to 11,950 (arbitrary units), for 6 and $20 \mu \mathrm{L}$, respectively. The test used to determine significant differences was a single factor ANOVA analysis, obtaining a level of signification (R) of 0.06 . In terms of signal reproducibility, the best results were obtained with 12,18 , and $20 \mu \mathrm{L}$ solutions (RSD below $12 \%)$. The larger volumes generate a drop that with time spread out occupying circular areas very close each other. For these reasons, $12 \mu \mathrm{L}$ sample solution was selected for further analysis, covering the whole array surface and preventing cross-contamination between flanking samples.

\subsection{Determination of matrix interference}

Food matrices can modify the response of the arrays on disc, eventually impeding an adequate quantification of target analytes. This type of interference is basically dependent on the commodity under consideration. In the case of fruit juices, matrix interference on competitive immunoassays can be overcome by simple dilution prior to analysis. Due to the assay sensitivity achieved in PBST ( IC $_{50}$ 1.9 and $1.6 \mu \mathrm{g} \mathrm{L}^{-1}$ for CLP and FTN, respectively), a dilution step did not compromise detectability level required for screening small organic pollutants such as pesticides in monitoring programs. To study the matrix effect on sensitivity, commercial mandarin juices were diluted 5, 10, 20 and $50(\mathrm{v} / \mathrm{v})$ fold in PBST. This study was performed using CLP as a model analyte. The diluted samples were used to prepare CLP standard curves, from 0.1 to $1000 \mathrm{mg} \mathrm{L}^{-1}$, in order to determine the extent of matrix interference. As it can be seen in Fig. 2, the $\mathrm{IC}_{50}$ of the majority of the assays ranged from 1.9 to $2.1 \mu \mathrm{gL}^{-1}$ (curves A, B, C and D). In contrary, IC 50 of the 5 fold diluted curve varied 50\% with respect to that obtained in PBST. From this study, it was found that any significant negative effects in terms of sensitivity and reproducibility ( $t$-student) could be observed after a simple 10 -fold dilution (curve $D$ ) in comparison to that obtained in working buffer (curve A). This is very important

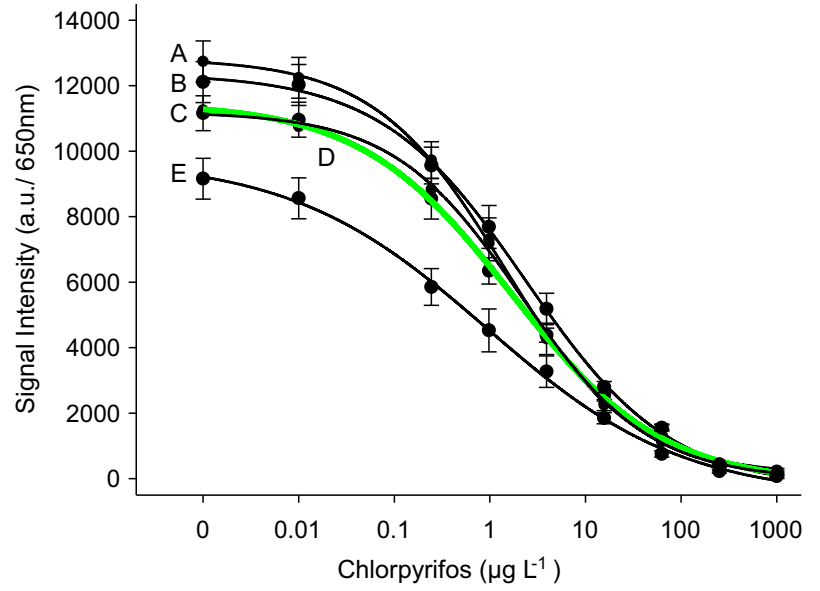

Fig. 2. Juice matrix interference on chlorpyrifos competitive assay. Standard curves are made in PBST (A), mandarin juice diluted 50 (B), 20 (C), 10 (D; curve in green) and 5 (E) fold in PBST. (For interpretation of the references to color in this figure legend, the reader is referred to the web version of this article.)

when transferring the method to industry that demands easy and practical sample treatment procedures.

\subsection{Multiplexed competitive assays}

Simultaneous determination of CLP and FTN was performed by an all-in-one reaction assay. For these experiments, specific single-target assays were integrated into one by preparing a unique mixed-sera solution. For that, the cocktail solution had the same sera concentration as that used in single-target assays. Signalto-noise ratios were $78.6 \pm 5.2$ and $39.4 \pm 3.3$ for CLP and FTN, respectively, being these values similar to those for the corresponding single-target assays (see page 8 ). In particular, $\mathrm{S} / \mathrm{N}$ variation ranged from $2.8 \%$ to $5.5 \%$, indicating an acceptable difference between single and multiplexed assays.

The calibration curves for duplex assay in 10 fold diluted mandarin juice are shown in Fig. 3.

The plots displayed the mean of 24 curves ( 3 discs) performed on different days, averaging a total of 96 spots per concentration of analyte. The intradisk RSD for all the analyte concentrations

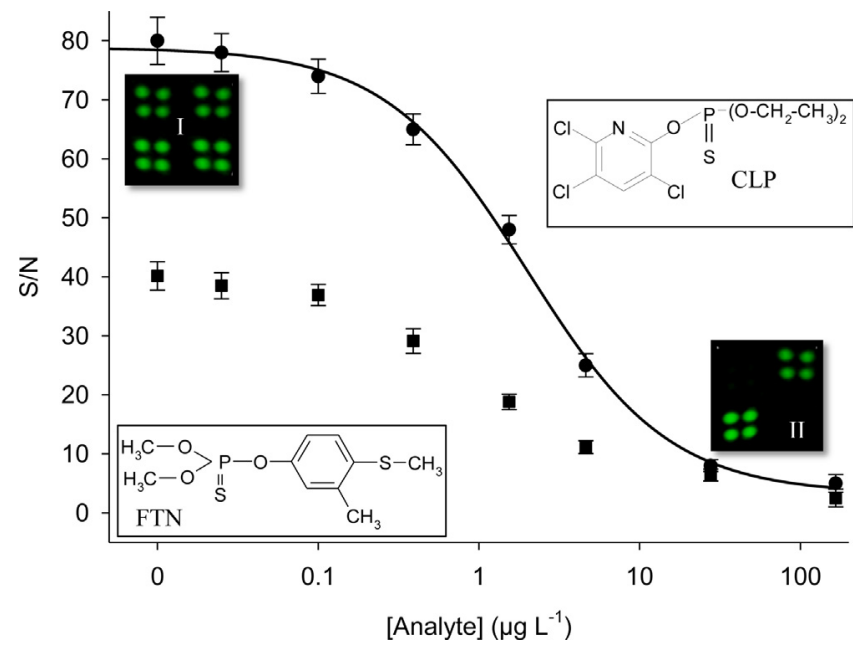

Fig. 3. Calibration curves of competitive multiplexed assays on discs for chlorpyrifos $(\mathrm{CLP}, \bullet)$ and fenthion (FTN, $\mathbf{a})$ in 10-fold diluted mandarin juice. Panels correspond to the images obtained in absence (panel I) and presence of $150 \mu \mathrm{g} \mathrm{L}^{-1}$ of analytes (panel II). The insets show the chemical structures of chlorpyrifos (CLP) and fenthion (FTN). 
Table 2

Determination of chemical residues in mandarin juice samples by duplex disc-arrays and GC-MS technique.

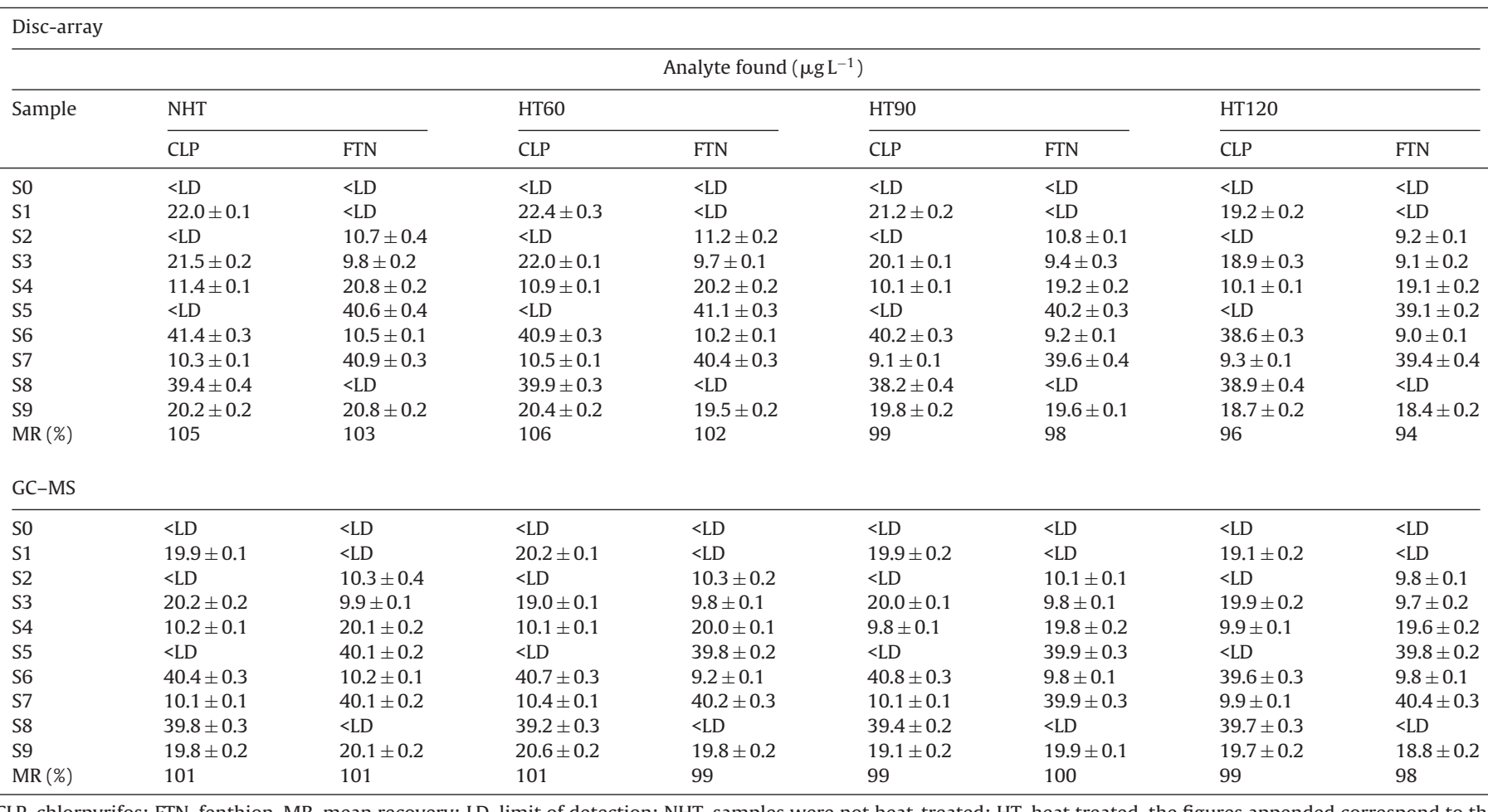

CLP, chlorpyrifos; FTN, fenthion. MR, mean recovery; LD, limit of detection; NHT, samples were not heat-treated; HT, heat treated, the figures appended correspond to the

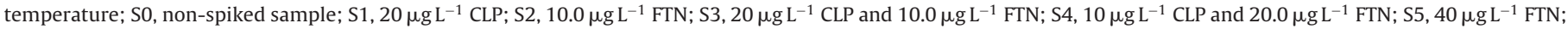

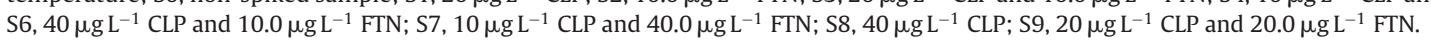

ranged from $6.3 \%$ to $8.6 \%$, while the mean interdisk RSD varied from $9.2 \%$ to $12.3 \%$. As analyte concentration increased, the signalacquired intensity varied from the highest (absence of analyte) to the background signal. The resulting silver deposit from the highest analyte concentration was minimal $(\mathrm{S} / \mathrm{N}<3)$, indicating a small degree of nonspecific response. The analytical performances measured as limit of detection, sensitivity $\left(\mathrm{IC}_{50}\right)$ and working interval were 0.2 and $0.1 \mu \mathrm{gL}^{-1}, 2.1$ and $1.5 \mu \mathrm{gL}^{-1}$, and $0.4-8.2$ and 0.2-6.3 $\mu \mathrm{g} \mathrm{L}^{-1}$, for CLP and FTN, respectively. No significant differences were detected for any of them in terms of sensitivity between the assay performed individually or duplexed and with that obtained by ELISA plate, using the same immunoreagents. In both cases, the high sensitivity reached for chlorpyrifos and fenthion allows for the quantitative analysis below the maximum EU residue limit for mandarin juice $\left(2 \mathrm{mg} \mathrm{L}^{-1}\right.$ for chlorpyrifos and $3 \mathrm{mg} \mathrm{L}^{-1}$ for fenthion).

\subsection{Mandarin juice sample analysis}

Commercial bottled juice samples were purchased from local markets of Valencia. Also, raw mandarins were bought and squeezed in the lab to obtain fresh juice samples. All samples were stored at $-80^{\circ} \mathrm{C}$ till the analysis.

Several methodologies have been tested for dissipation of pesticide residues in citrus concentrates [22]; however the effect of heat treatment on pesticide dissipation in mandarin juice, to the best of our knowledge, has not been reported. For this study, ten samples of $100 \mathrm{~mL}$ of natural and commercial juices were spiked at different levels within the working interval with known amounts of CLP and FTN $\left(10,20\right.$ and $\left.40 \mu \mathrm{g} \mathrm{L}^{-1}\right)$. Before spiking, samples were analyzed by GC-MS for incurred chlorpyrifos or fenthion residues and confirmed that were free of pesticide contamination. To study the effect of thermal treatment on pesticide dissipation, samples were heated at 60,90 and $120^{\circ} \mathrm{C}$ for $60 \mathrm{~s}$. Then, the samples were left for $20 \mathrm{~min}$ to reach room temperature. Before analysis, samples were conditioned by adding the appropriate volume of sera cocktail and working buffer solution and then, the duplex assay was performed as described. Each sample was also analyzed by triplicate on the same day and on the same disc to verify reproducibility and minimize pesticide degradation. Finally, the disk was read by the DVD drive, obtaining quantitative results. As is shown in Table 2, recovery intervals were $96-105 \%$ and $94-103 \%$, for chlorpyrifos and fenthion respectively. These results are in good agreement with those obtained by GC-MS. Accordingly, the developed duplex arrays on disc provided results that were statistically comparable to those obtained by GC-MS for the analysis of mandarin juices at trace levels by simple dilution without filtration.

Regarding the effect of heat treatment, it might be concluded that the behavior of both pesticides is similar. Dissipation of pesticide residues from juice matrix under the studied heat treatments is insignificant. These results are very important since the proposed methodology can be a suitable methodology to analyze agrochemical residues on both unprocessed and processed mandarin juice samples.

\section{Conclusions}

To the best of our knowledge, this is the first example of using arrays on disc in combination with a standard DVD drive for quantification, in a selective manner, pesticide residues in juice samples at $\mu \mathrm{g} \mathrm{L}^{-1}$. Results of optimization experiments demonstrated that a simple sample dilution eliminates matrix interferences, without compromising the detectability level required for the determination of agrochemical residues below the established maximum residue levels. Compact disc-based methodology is user-friendly (no chemical solvents are required), affordable and robust, with 
high applicability to in situ analysis and monitoring programs. The capability for analyzing large number of samples; in this disc configuration 64 juices can be simultaneously tested; provides results in a quick way ( $40 \mathrm{~min}$ ) at a competitive value. Also, the low volume of sample required in combination with the good assay sensitivity and selectivity make this methodology very promising for other applications where sample is limited such as in diagnostics and clinical chemistry. In summary, arrays on disc as platform and DVD drives as chemical detector is a powerful and cost-effective analytical tool with high potential for screening applications. Also, it might be of great interest in routine testing for food safety and quality control, ensuring similar results in terms of sensitivity, selectivity and reproducibility to reference methodologies.

\section{Acknowledgments}

This work was funded by the projects FEDER CTQ2010-15943 (CICYT, Spain), and PROMETEO 2010/008 and ACOMP/2012/158 (Generalitat Valenciana).

\section{References}

[1] C. Lu, F.J. Schenck, M.A. Pearson, J.W. Wong, Environ. Health Perspect. 118 (2010) 1625-1630.

[2] European Food Safety Authority, The 2009 European union report on pesticide residues in food, EFSA J. 9 (11)(2011) 2430
[3] B.A. Denninson, J. Am. Coll. Nutr. 15 (1996) 4S-11S.

[4] M.A. Aramendia, V. Borau, F. Lafont, A. Marinas, J.M. Marinas, J.M. Moreno, F.J. Urbano, Food Chem. 105 (2007) 855-865.

[5] J. Fenik, M. Tankiewicz, M. Biziuk, TrAC 30 (2011) 814-826.

[6] Y. Pico, M. Farre, C. Soler, D. Barceló, Anal. Chem. 79 (2007) 9350-9363.

[7] P.D. Patel, TrAC 21 (2002) 96-115.

[8] S.R. Raz, W. Haasnoot, TrAC 30 (2011) 1526-1537.

[9] C.R. Suri, R. Boro, Y. Nangia, S. Gandhi, P. Sharma, N. Wangoo, K. Rajesh, G.S. Shekhawat, TrAC 28 (2009) 29-39.

[10] G.A. Posthuma-Trumpie, J. Korf, A. van Amerongen, Anal. Bioanal. Chem. 393 (2009) 569-582.

[11] C. Liu, Q. Jia, C. Yang, R. Qiao, L. Jing, L. Wang, C. Xu, M. Gao, Anal. Chem. 83 (2011) 6778-6784

[12] G. Zhou, X. Mao, D. Juncker, Anal. Chem. 84 (2012) 7736-7743.

[13] I. Guillén, J.A. Gabaldón, E. Núñez-Delicado, R. Puchades, A. Maquieira, S. Morais, Food Chem. 129 (2011) 624-629.

[14] M. Blazkova, B. Micova-Holubova, P. Rauch, L. Fukal, Biosens. Bioelectron. 25 (2009) 753-758.

[15] G. MacBeath, Nat. Genet. 35 (2002) 526-532.

[16] L. Wang, P.C.H. Li, Anal. Chim. Acta 687 (2011) 12-27.

[17] Z. Zhang, P. Li, X. Hu, Q. Zhang, X. Ding, W. Zhang, Sensors 12 (2012) 9234-9252.

[18] T. Arnandis-Chover, S. Morais, L.A. Tortajada-Genaro, R. Puchades, A. Maquieira, G. Olabarria, J. Berganza, Talanta 101 (2012) 405-412.

[19] S. Morais, J. Tamarit-López, R. Puchades, A. Maquieira, Environ. Sci. Technol. 44 (2010) 9024-9029.

[20] M. Garcés-García, E.M. Brun, R. Puchades, A. Maquieira, Anal. Chim. Acta 556 (2006) 347-354.

[21] S. Morais, J. Tamarit-López, J. Carrascosa, R. Puchades, A. Maquieira, Anal. Bioanal. Chem. 391 (2008) 2837-2844.

[22] B.M. Keikotlhaile, P. Spanoghe, W. Steurbaut, Food Chem. Toxicol. 48 (2010) $1-6$ 